\title{
Existence and Regularity of Solutions for Unbounded Elliptic Equations with Singular Nonlinearities
}

\author{
Aziz Bouhlal $\mathbb{1}^{1}$ and Jaouad Igbida $\mathbb{D}^{2}$ \\ ${ }^{1}$ Laboratoire de Mathématiques et Applications, Faculty of Sciences, B.P.20, El Jadida, Morocco \\ ${ }^{2}$ Labo DGTIC, Department of Mathematics, CRMEF, El Jadida, Morocco \\ Correspondence should be addressed to Aziz Bouhlal; a.bouhlal86@gmail.com
}

Received 19 January 2021; Revised 4 April 2021; Accepted 19 April 2021; Published 27 April 2021

Academic Editor: Jaume Giné

Copyright (c) 2021 Aziz Bouhlal and Jaouad Igbida. This is an open access article distributed under the Creative Commons Attribution License, which permits unrestricted use, distribution, and reproduction in any medium, provided the original work is properly cited.

For $q, \gamma>0$, we study existence and regularity of solutions for unbounded elliptic problems whose simplest model is $\left\{\begin{array}{ll}-\operatorname{div}\left[\left(1+|u|^{q}\right) \nabla u\right]=\left(f /|u|^{c}\right) & \text { in } \Omega \\ u=0 & \text { on } z \Omega\end{array}\right.$, where $f \in L^{m}(\Omega), m \geq 1$.

\section{Introduction}

Consider the Dirichlet problem for some nonlinear elliptic equations:

$$
-\operatorname{div}\left(\left[a(x)+|u|^{q}\right] \nabla u\right)=\frac{f}{|u|^{\gamma}}, \quad x \in \Omega, u \in H_{0}^{1}(\Omega),
$$

under the following assumptions. The set $\Omega$ is a bounded open subset of $\mathbb{R}^{N}$, with $N \geq 3$ :

$$
q, \gamma>0
$$

$a: \Omega \longrightarrow \mathbb{R}$ is a measurable function satisfying the following conditions:

$$
\alpha \leq a(x) \leq \beta
$$

for almost every $x \in \Omega$, where $\alpha$ and $\beta$ are positive constant, and

$$
0 \leqq f \in L^{m}(\Omega), \quad \text { with } m \geq 1 .
$$

A possible motivation for studying the existence of these types of problems arises from the calculation of variations and stochastic control. For example, if we consider the functional

$$
J(v)=\frac{1}{2} \int_{\Omega}\left[a(x)+|v|^{1-\theta}\right]|\nabla v|^{2}-\int_{\Omega} f(x) v,
$$

the Euler-Lagrange equation associated to the functional $J$ is

$$
-\operatorname{div}\left(\left[a(x)+|v|^{1-\theta}\right] \nabla v\right)+\frac{1-\theta}{2} \frac{|\nabla v|^{2}}{|v|^{\theta}} \operatorname{sign}(v)=f .
$$

Several papers deal with existence of solutions to the singular elliptic problems with lower order terms having a quadratic growth with respect to the gradient (for example, [1-9]), namely, with the model problem

$$
\begin{cases}-\operatorname{div}(M(x, u) \nabla u)+\frac{|\nabla u|^{2}}{|u|^{\theta}} \operatorname{sign}(u)=f(x), & x \in \Omega, \\ u(x)=0, & x \in \partial \Omega,\end{cases}
$$

where $\theta$ is a positive constant and $M: \Omega \times \mathbb{R} \longrightarrow \mathbb{R}$ is a Carathéodory function. More precisely, existence of positive solutions for (7) was shown in [1-3], for $M(x, t)=1$ and $0<\theta \leq 1$, and the uniqueness of positive solution, for $M(x, t)=1$ and $0<\theta<1$, in [4]. On the contrary, the existence of positive solutions of (7) is shown in [6] for $0<\theta \leq 1$, provided $M$ is a bounded uniformly elliptic matrix and $0 \supsetneqq f \in L^{m}(\Omega)(m>(2 N / N+2))$. Later, in [9], it is 
proved the existence of solution for (7) with $0<\theta<1$, where $M(x, t)=1$ and the data $f \in L^{m}(\Omega)$ with $m>(N / 2)$, and does not satisfy any sign assumption. Recently, a problem introduced by L. Boccardo (see $[7,10]$ ) has given a strong impulse to the study of quasilinear problems having the unbounded divergence operator. In particular, in [7], the authors have proved the existence of positive solutions to problem (7) under the assumption that $0<\theta<1$, $M(x, t)=1+|t|^{q}$, and $0 \supsetneqq f \in L^{m}(\Omega)$. We refer also that, in [5], the author has shown the same result as in [7], in the case $0<\theta<1$ and without any sign restriction over $f$.

Let us now consider the Dirichlet boundary value problem (7) in the simple case:

$$
\begin{cases}-2 \Delta u+\frac{|\nabla u|^{2}}{u}=f(x), & x \in \Omega, \\ u(x)=0, & x \in \partial \Omega .\end{cases}
$$

If we define $v=2(u / \sqrt{|u|})$, then the function $v$ is solution of

$$
\begin{cases}-\Delta v=\frac{f(x)}{|v|}, & x \in \Omega, \\ v(x)=0, & x \in \partial \Omega,\end{cases}
$$

which is singular on the right-hand side. Let us remark that, in the case of nonnegative $f$, in [11], the authors considered the elliptic semilinear problems whose model is

$$
\begin{cases}-\Delta u=\frac{f}{u^{\gamma}}, & x \in \Omega, \\ u=0, & x \in \partial \Omega,\end{cases}
$$

where $\gamma>0$. More precisely, they have shown that the term $\left(f /|u|^{\gamma}\right)$ has a regularizing effect on the solutions $u$. In [12], the author has shown the existence of solutions to the following elliptic problem with degenerate coercivity:

$$
\begin{cases}-\operatorname{div}\left(\frac{\nabla u}{(1+|u|)^{p}}\right)=\frac{f}{|u|^{\gamma}}, & x \in \Omega, \\ u=0, & x \in \partial \Omega,\end{cases}
$$

where $p, \gamma>0$.

The purpose of this paper is to study the same kind of lower order term as in problems (7) and (9) (indeed, $\left.\left(f /|u|^{\gamma}\right)\right)$ in the case of an elliptic operator with unbounded coefficients. The main difficulties posed by this problem were that the principal part of the differential operator $\operatorname{div}\left(\left(a(x)+|u|^{q}\right) \nabla u\right)$ is not well defined on the whole $H_{0}^{1}(\Omega)$; the solutions did not belong, in general, to $H_{0}^{1}(\Omega)$ and the lower order term has a singularity at $u=0$. Despite these difficulties, we prove that, in our case too, the lower order term $\left(f /|u|^{\gamma}\right)$ has a regularizing effect.

Our main existence results are as follows.
Theorem 1. Assume that (2) and (3) hold true. If $0 \lessgtr f \in L^{m}(\Omega)$ with $m>(N / 2)$, then there is a positive solution $u \in L^{\infty}(\Omega)$ of (1), in the sense of distributions, that is,

$$
\int_{\Omega}\left[a(x)+u^{q}\right] \nabla u \nabla \varphi=\int_{\Omega} \frac{f \varphi}{u^{\gamma}},
$$

for any test function $\varphi$ in $C_{0}^{1}(\Omega)$. Moreover, we have the following summability results for $u$ :

(1) Let $0<q<1$ :

(i) If $0<\gamma \leq 1-q$, then $u \in H_{0}^{1}(\Omega)$.

(ii) If $\gamma>1-q$, then $u \in H_{l o c}^{1}(\Omega)$.

(2) Let $q=1$ :

(i) If $0<\gamma \leq 1$, then $u \in H_{0}^{1}(\Omega)$.

(ii) If $\gamma>1$, then $u \in H_{l o c}^{1}(\Omega)$.

(3) Let $q>1$, then $u \in H_{l o c}^{1}(\Omega)$.

When $f \in L^{m}(\Omega), \quad 1<m<(N / 2)$, we will prove the following regularizing effects.

Theorem 2. We suppose that $0 \supsetneqq f \in L^{m}(\Omega), 1<m<(N / 2)$ and that (2) and (3) are satisfied. If $0<q<1$, then, there exists a solution $u$ of (1) in the sense (19), such that

(1) If $\gamma<1-q$ and $(2 * / 2 *+q-1+\gamma) \leq m<(N / 2)$, then $u \in H_{0}^{1}(\Omega) \cap L^{m^{* *}(1+q+\gamma)}(\Omega)$, where

$$
m^{* *}=\left(m^{*}\right)^{*}=\frac{N m}{N-2 m} .
$$

(2) If $\gamma=1-q$, then $u \in H_{0}^{1}(\Omega)$.

(3) If $\gamma>1-q$, then $u \in L^{(1+q+\gamma / 2) 2^{*}}(\Omega) \cap H_{l o c}^{1}(\Omega)$.

Notation: throughout this paper, we fix an integer $N \geq 3$. For any $p>1, p^{\prime}=(p / p-1)$ will be the Hölder conjugate exponent of $p$, and if $1 \leq p<N$, we will denote by $p^{*}=$ $(N p / N-p)$ the Sobolev conjugate exponent of $p$. As usual, let us denote by $\mathcal{S}$ the Sobolev constant, i.e.,

$$
\mathcal{S}=\inf _{\left.u \in H_{0}^{1}(\Omega) \dashv 0\right\}} \frac{\|\nabla u\|_{2}^{2}}{\|u\|_{2^{*}}^{2}} .
$$

We denote by $\mathscr{P}$ the Poincaré constant given by

$$
\mathscr{P}=\inf _{u \in H_{0}^{1}(\Omega)-\{0\}} \frac{\|\nabla u\|_{2}^{2}}{\|u\|_{2}^{2}} .
$$

For all $k>0$, we recall the definition of a truncated function $T_{k}(s)$ defined by

$$
T_{k}(s)=\max \{\min \{k, s\}-k\} .
$$

We also consider

$$
G_{k}(s)=s-T_{k}(s)
$$

As usual, we consider the positive and negative part of a measurable function $u(x)$ 


$$
\begin{aligned}
u(x) & =u^{+}(x)-u^{-}(x), \quad \text { where } u^{+}(x) \\
& =u(x) \chi_{\{u \geq 0\}} \text { and } u^{-}(x)=-u(x) \chi_{\{u<0\}} .
\end{aligned}
$$

\section{The Approximated Problem}

To prove our existence results, we will use the following approximating problems:

$$
-\operatorname{div}\left(\left[a(x)+\left|u_{n}\right|^{q}\right] \nabla u_{n}\right)=\frac{f_{n}}{\left(\left|u_{n}\right|+(1 / n)\right)^{\gamma}}, \quad x \in \Omega,
$$

where $n \in \mathbb{N}^{*}$, and

$$
f_{n}(x)=\frac{f(x)}{1+(1 / n)|f(x)|}
$$

As in [11], we prove existence of positive solution of the approximated problem.

Lemma 1. Let $g$ be positive function belonging to $L^{\infty}(\Omega)$. Suppose that (2) and (3) are satisfied. Then, there exists a positive solution $u_{n} \in H_{0}^{1}(\Omega) \cap L^{\infty}(\Omega)$ of the problem

$$
-\operatorname{div}\left(\left[a(x)+\left|u_{n}\right|^{q}\right] \nabla u_{n}\right)=\frac{g}{\left(\left|u_{n}\right|+(1 / n)\right)^{\gamma}}, \quad x \in \Omega, u_{n} \in H_{0}^{1}(\Omega) .
$$

Proof. To prove it, we define the following operator $S_{n}: L^{2}(\Omega) \longrightarrow L^{2}(\Omega)$ which associates to every $v \in L^{2}(\Omega)$ the solution $w_{n} \in H_{0}^{1}(\Omega)$ to

$$
\begin{cases}-\operatorname{div}\left(\left[a(x)+\left|T_{n}\left(w_{n}\right)\right|^{q}\right] \nabla w_{n}\right)=\frac{g}{(|v|+(1 / n))^{\gamma},} & \text { in } \Omega, \\ w_{n}=0, & \text { on } \partial \Omega .\end{cases}
$$

From the results of [13], the operator $S_{n}$ is well defined and $w_{n}$ is bounded by the results of [14]. We take $w_{n}$ as a test function in (19), and we use Hölder's inequality and (3) to deduce that

$$
\begin{aligned}
\alpha \int_{\Omega}\left|\nabla w_{n}\right|^{2} & \leq \int_{\Omega}\left[a(x)+\left|T_{n}\left(w_{n}\right)\right|^{q}\right]\left|\nabla w_{n}\right|^{2}=\int_{\Omega} \frac{g w_{n}}{(|v|+(1 / n))^{\gamma}} \\
& \leq n^{\gamma}\|g\|_{L^{\infty}(\Omega)} \int_{\Omega}\left|w_{n}\right| \\
& \leq n^{\gamma}\|g\|_{L^{\infty}(\Omega)} \sqrt{|\Omega|}\left\|w_{n}\right\|_{L^{2}(\Omega)}
\end{aligned}
$$

Thanks to Poincaré's inequality, we deduce

$$
\alpha \mathscr{P}\left\|w_{n}\right\|_{L^{2}(\Omega)}^{2} \leq n^{\gamma}\|g\|_{L^{\infty}(\Omega)} \sqrt{|\Omega|}\left\|w_{n}\right\|_{L^{2}(\Omega)} .
$$

Hence, there exists an invariant ball for $S_{n}$. On the contrary, from the $H_{0}^{1}(\Omega) \hookrightarrow L^{2}(\Omega)$ embedding, it is easily seen that $S_{n}$ is continuous and compact. The Schauder theorem shows that $S_{n}$ has a fixed point or equivalently, and there exists a solution $u_{n} \in H_{0}^{1}(\Omega)$ to problems

$$
\begin{cases}-\operatorname{div}\left(\left[a(x)+\left|T_{n}\left(u_{n}\right)\right|^{q}\right] \nabla u_{n}\right)=\frac{g}{\left(\left|u_{n}\right|+(1 / n)\right)^{\gamma},} & \text { in } \Omega, \\ u_{n}=0, & \text { on } \partial \Omega .\end{cases}
$$

Moreover, by the maximum principle, it is clear that the sequence $u_{n}$ is nonnegative since $g$ is nonnegative, and we choose $G_{k}\left(u_{n}\right)$ as test function in (25) and use (3) to obtain

$$
\alpha \int_{A_{k}}\left|G_{k}\left(u_{n}\right)\right|^{2} \leq \frac{1}{k^{\gamma}} \int_{A_{k}} g G_{k}\left(u_{n}\right),
$$

where $A_{k}=\left\{x \in \Omega:\left|u_{n}\right|>k\right\}$. By the method of Stampacchia (see [14]), the sequence $u_{n}$ is bounded in $L^{\infty}(\Omega)$. Supposing that $u_{n}$ is bounded by $d_{n}$ in $L^{\infty}(\Omega)$, we have that $u_{n}$ : = $u_{n+\left[d_{n}\right]+1} \in L^{\infty}(\Omega) \cap H_{0}^{1}(\Omega)$ is a solution of $(13)$.

By Lemma 1, it follows the existence of a solution $u_{n} \in L^{\infty}(\Omega) \cap H_{0}^{1}(\Omega)$ of $(19)$.

Now, we are going to prove that the sequence $u_{n}$ is not 0 in $\Omega$. For this, we are going to prove that it is uniformly away from zero in every compact set in $\Omega$. We will follow a similar technique to that one in [12].

Lemma 2. Assume that (2) and (3) hold true. If $0 \leqq f \in L^{1}(\Omega)$ and $u_{n}$ is the solution of problem (19), then for every $n \in \mathbb{N}^{*}$ : $u_{n} \leq u_{n+1}$ a.e. in $\Omega$. Furthermore, if $\omega \subset \subset \Omega$, then, for every $n \in \mathbb{N}^{*}$, there exists $c_{\omega}>0$ such that $u_{n} \geq c_{\omega}>0$ a.e. in $\omega$.

Proof. Let us consider $T_{k}\left[\left(u_{n}-u_{n+1}\right)^{+}\right]$as a test function in problems (19). Then,

$$
\int_{\Omega}\left[a(x)+u_{n}^{q}\right] \nabla u_{n} \nabla T_{k}\left[\left(u_{n}-u_{n+1}\right)^{+}\right]=\int_{\Omega} \frac{f_{n}}{\left(\left|u_{n}\right|+(1 / n)\right)^{\gamma}} T_{k}\left[\left(u_{n}-u_{n+1}\right)^{+}\right] .
$$


Observing that $f_{n} \leq f_{n+1}$, we have

$$
\begin{aligned}
\int_{\Omega} \frac{f_{n}}{\left(u_{n}+(1 / n)\right)^{\gamma}} T_{k}\left[\left(u_{n}-u_{n+1}\right)^{+}\right] & \leq \int_{\Omega} \frac{f_{n+1}}{\left(u_{n+1}+(1 / n+1)\right)^{\gamma}} T_{k}\left[\left(u_{n}-u_{n+1}\right)^{+}\right] \\
& =\int_{\Omega}\left[a(x)+u_{n+1}^{q}\right] \nabla u_{n+1} \nabla T_{k}\left[\left(u_{n}-u_{n+1}\right)^{+}\right] \\
& \leq \int_{\Omega}\left[a(x)+u_{n}^{q}\right] \nabla u_{n+1} \nabla T_{k}\left[\left(u_{n}-u_{n+1}\right)^{+}\right] .
\end{aligned}
$$

Therefore, by (3), we deduce that

$$
\alpha \int_{\Omega}\left|\nabla T_{k}\left[\left(u_{n}-u_{n+1}\right)^{+}\right]\right|^{2} \leq \int_{\Omega}\left[a(x)+u_{n}^{q}\right]\left|\nabla T_{k}\left[\left(u_{n}-u_{n+1}\right)^{+}\right]\right|^{2} \leq 0 .
$$

Consequently, we obtain $\int_{\Omega}\left|\nabla T_{k}\left[\left(u_{n}-u_{n+1}\right)^{+}\right]\right|^{2}=0$, so by Poincaré's inequality, we have $T_{k}\left[\left(u_{n}-u_{n+1}\right)^{+}\right]=0$ for every $k>0$. Thus, $u_{n} \leq u_{n+1}$ a.e. $x \in \Omega$.

We remark that $u_{1}$ is bounded; indeed, $\left|u_{1}\right| \leq c$, for some positive constant $c$. Then, it follows that

$$
-\operatorname{div}\left(\left[a(x)+\left|u_{1}\right|^{q}\right] \nabla u_{1}\right) \geq \frac{f_{1}}{(c+1)^{\gamma}}, \quad x \in \Omega .
$$

Thanks to (3), we have $\alpha \leq a(x)+\left|u_{1}\right|^{q} \leq \beta+c^{q}$. Thus, we infer that $u_{1}$ is a supersolution of a linear Dirichlet problem with a strictly positive and bounded, measurable coefficient. The strong maximum principle implies that $u_{1}>0$. In addition, Harnack's inequality gives the stronger conclusion: for every $\omega \subset \subset \Omega$, there exists $c_{\omega}$ such that $u_{1} \geq c_{\omega}$ a.e. in $\omega$. Finally, using that the sequence $u_{n}$ is increasing, one deduces that $u_{n} \geq c_{\omega}$ a.e. in $\omega$ for every $n \in \mathbb{N}^{*}$.

2.1. Existence of Bounded Solutions. In this section, we will prove existence of bounded weak solutions for (1).

Lemma 3. Let $0 \lessgtr f \in L^{m}(\Omega)$ with $m>(N / 2)$. Suppose that (2) and (3) hold true. Let $\left\{u_{n}\right\}$ be a sequence solutions of (19) with $f_{n}=f$ for every $n \in \mathbb{N}^{*}$. Then, the norm of the sequence $\left\{u_{n}\right\}$ in $L^{\infty}(\Omega)$ is bounded by a constant which depends on $q, m, N, \alpha, \gamma$, meas $(\Omega)$ and on the norm of $f$ in $L^{m}(\Omega)$.
Proof. The use of $G_{k}\left(u_{n}\right)$ as test function in (19) and (3), implies that

$$
\alpha \int_{A_{k}}\left|\nabla G_{k}\left(u_{n}\right)\right|^{2} \leq \frac{1}{k^{\gamma}} \int_{A_{k}} f G_{k}\left(u_{n}\right),
$$

where $A_{k}=\left\{x \in \Omega:\left|u_{n}\right|>k\right\}$. Hence, we can use Theorem 4.1 in [14] and obtain a positive constant, say $M$, that only depends on the parameters: $q, N, \alpha, \gamma$, meas $(\Omega)$ and $\|f\|_{L^{m}(\Omega)}$ such that: $\left\|u_{n}\right\|_{L^{\infty}(\Omega)} \leq M$ for all $n \in \mathbb{N}^{*}$.

Lemma 4. We assume that $0 \supsetneqq f \in L^{m}(\Omega)$ with $m>(N / 2)$, and (2) and (3) are satisfied. Let $\left\{u_{n}\right\}$ be a sequence solutions of (19) with $f_{n}=f$ for every $n \in \mathbb{N}^{*}$. If $q<1$ and $\gamma \leq 1-q$, then the sequence $\left\{u_{n}\right\}$ is uniformly bounded in $H_{0}^{1}(\Omega)$.

Proof. We denote by $C$ a positive constant which may only depend on the parameters of our problem, and its value may vary from line to line.

We use $\left(1+u_{n}\right)^{1-q}-1$ as test function in (19) to obtain

$$
(1-q) \int_{\Omega} \frac{a(x)+u_{n}^{q}}{\left(1+u_{n}\right)^{q}}\left|\nabla u_{n}\right|^{2} \leq C \int_{\Omega} f\left|u_{n}\right|^{1-q-\gamma},
$$

and thus (since $q \leq 1$ ),

$$
(1-q) \min (\alpha, 1) \int_{\Omega}\left|\nabla u_{n}\right|^{2} \leq C \int_{\Omega}\left|f\left\|\left|u_{n}\right|^{1-q-\gamma} \leq C\right\| u \|_{n} L_{\infty(\Omega)}^{1-q-\gamma} \int_{\Omega} f \leq C .\right.
$$

from which the sequence $u_{n}$ is bounded in $H_{0}^{1}(\Omega)$. 
Lemma 5. Let $0 \lessgtr f \in L^{m}(\Omega)$ with $m>(N / 2)$, and we suppose that (2) and (3) are satisfied. If $q<1$ and $\gamma>1-q$ and $u_{n}$ is a solution to problem (19), then $u_{n}$ is uniformly bounded in $H_{l o c}^{1}(\Omega)$.
Proof. Let $\varphi \in C_{0}^{1}(\Omega)$ and $\omega=\operatorname{Supp} \varphi$ be the support of $\varphi$; then, from Lemma 2 , there exists $c_{\omega}>0$ such that $u_{n} \geq c_{\omega}$ for a.e. $x \in \omega$.

Choosing $\left[\left(u_{n}+1\right)^{1-q}-1\right] \varphi^{2}$ as test function in (19) and using (3), we obtain

$$
\begin{array}{r}
\alpha(1-q) \int_{\Omega}\left|\nabla u_{n}\right|^{2} \varphi^{2}+2 \int_{\Omega}\left[a(x)+u_{n}^{q}\right]\left[\left(u_{n}+1\right)^{1-q}-1\right] \nabla u_{n} \nabla \varphi \varphi \\
\leq \int_{\Omega} \frac{f_{n}}{\left(u_{n}+(1 / n)\right)^{\gamma}}\left[\left(u_{n}+1\right)^{1-q}-1\right] \varphi^{2} \leq \frac{\|\varphi\|_{L^{\infty}(\Omega)}^{2}}{c_{\omega}^{\gamma}} \int_{\Omega} f,
\end{array}
$$

which then implies

$$
\begin{aligned}
& \alpha(1-q) \int_{\Omega}\left|\nabla u_{n}\right|^{2} \varphi^{2} \\
& \leq \frac{\|\varphi\|_{L^{\infty}(\Omega)}^{2}}{c_{\omega}^{\gamma}} \int_{\Omega} f-2 \int_{\Omega}\left[a(x)+u_{n}^{q}\right]\left[\left(u_{n}+1\right)^{1-q}-1\right] \nabla u_{n} \nabla \varphi \varphi .
\end{aligned}
$$

Using (3), we have

$$
a(x)+t^{q} \leq c_{0}(1+t)^{q},
$$

for every $q>0$ and $t \geq 0$ (and for a suitable $c_{0}$ independent on $n)$.

We then have

We can use Young's inequality with $\epsilon$, and we obtain

$$
\begin{aligned}
& 2\left|\int_{\Omega}\left[a(x)+u_{n}^{q}\right]\left[\left(u_{n}+1\right)^{1-q}-1\right] \nabla u_{n} \nabla \varphi \varphi\right| \\
& \leq \varepsilon \int_{\Omega}\left|\nabla u_{n}\right|^{2} \varphi^{2}+C(\varepsilon) \int_{\Omega}\left[a(x)+u_{n}^{q}\right]^{2}\left[\left(u_{n}+1\right)^{1-q}-1\right]^{2}|\nabla \varphi|^{2} .
\end{aligned}
$$

$$
2\left|\int_{\Omega}\left[a(x)+u_{n}^{q}\right]\left[\left(u_{n}+1\right)^{1-q}-1\right] \nabla u_{n} \nabla \varphi \varphi\right| \leq \varepsilon \int_{\Omega}\left|\nabla u_{n}\right|^{2} \varphi^{2}+C(\varepsilon) c_{0}^{2} \int_{\Omega} u_{n}^{2}|\nabla \varphi|^{2} .
$$

Applying (38) to (35) and letting $\varepsilon=(\alpha(1-q) / 2)$, we obtain

$$
\int_{\Omega}\left|\nabla u_{n}\right|^{2} \varphi^{2} \leq C+C \int_{\Omega} u_{n}^{2}|\nabla \varphi|^{2} \leq C+C\left\|u_{n}\right\|_{L^{\infty}(\Omega)}^{2} \int_{\Omega}|\nabla \varphi|^{2} \leq C,
$$

and this gives that $u_{n}$ is bounded in $H_{\text {loc }}^{1}(\Omega)$.

Lemma 6. Let $q=1$. Suppose that (2) and (3) hold. If $0 \leqq f \in L^{m}(\Omega)$ with $m>(N / 2)$, then the sequence $\left\{u_{n}\right\}$ defined by (19) satisfies the following summability:

(1) If $0<\gamma \leq 1$, then $u_{n}$ is uniformly bounded in $H_{0}^{1}(\Omega)$

(2) If $\gamma>1$, then $u_{n}$ is uniformly bounded in $H_{l o c}^{1}(\Omega)$
Proof. (1) Let us take $\log \left(1+u_{n}\right)$ as test function in (19) and use (3) to obtain that

$$
\begin{gathered}
\min (1, \alpha) \int_{\Omega}\left|\nabla u_{n}\right|^{2} \leq \int_{\Omega} f \frac{\log \left(1+u_{n}\right)}{\left(u_{n}+(1 / n)\right)^{\gamma}} \leq \int_{\Omega} f u_{n}^{1-\gamma} \\
\leq\left\|u_{n}\right\|_{L^{\infty}(\Omega)}^{1-\gamma} \int_{\Omega} f \leq C .
\end{gathered}
$$


(2) Let $\varphi \in C_{0}^{1}(\Omega)$ and choose $\log \left(1+u_{n}\right) \varphi^{2}$, as a test function in problem (19). From assumption (19), one has

$$
\begin{array}{r}
\min (1, \alpha) \int_{\Omega}\left|\nabla u_{n}\right|^{2} \varphi^{2}+2 \int_{\Omega}\left[a(x)+u_{n}^{q}\right] \log \left(1+u_{n}\right) \nabla u_{n} \nabla \varphi \varphi \\
\leq \int_{\Omega} f \frac{\log \left(1+u_{n}\right)}{\left(u_{n}+(1 / n)\right)^{\gamma}} \varphi^{2} \leq \int_{\Omega} f \frac{\varphi^{2}}{u_{n}^{\gamma-1}} \leq \frac{\|\varphi\|_{L^{\infty}}^{2}(\Omega)}{c_{\omega}^{\gamma-1}} \int_{\Omega} f,
\end{array}
$$

where $\omega=\operatorname{Supp} \varphi$. By Young's inequalities, it is easy to prove $2\left|\int_{\Omega}\left[a(x)+u_{n}^{q}\right] \log \left(1+u_{n}\right) \nabla u_{n} \nabla \varphi \varphi\right| \leq \varepsilon \int_{\Omega}\left|\nabla u_{n}\right|^{2} \varphi^{2}+C(\varepsilon)$.
Hence, equality (41) implies that

$\min (1, \alpha) \int_{\Omega}\left|\nabla u_{n}\right|^{2} \varphi^{2} \leq \frac{\|\varphi\|_{L^{\infty}(\Omega)}^{2}}{c_{\omega}^{\gamma-1}} \int_{\Omega} f+\varepsilon \int_{\Omega}\left|\nabla u_{n}\right|^{2} \varphi^{2}+C(\varepsilon)$.

Letting $\varepsilon=(\min (1, \alpha) / 2)$, we get that $u_{n}$ is bounded in $H_{\mathrm{loc}}^{1}(\Omega)$.

Lemma 7. Let $q>1$. Assume that (2) and (3) hold true. If $0 \nsupseteq f \in L^{m}(\Omega)$ with $m>(N / 2)$, then the solution $u_{n}$ of (19) is uniformly bounded in $H_{l o c}^{1}(\Omega)$.

Proof. Let $\varphi$ be a function in $C_{0}^{1}(\Omega)$ and $\omega=\operatorname{Supp} \varphi$. Take $\left[1-\left(u_{n}+1\right)^{1-q}\right] \varphi^{2}$ as test function in (19) and use (3) to obtain

$$
\begin{aligned}
\frac{\min (1, \alpha)}{2^{q-1}} \int_{\Omega}\left|\nabla u_{n}\right|^{2} \varphi^{2} & \leq(q-1) \min (1, \alpha) \int_{\Omega} \frac{1+u_{n}^{q}}{\left(1+u_{n}\right)^{q}}\left|\nabla u_{n}\right|^{2} \varphi^{2} \\
& \leq \int_{\Omega} \frac{f}{\left(u_{n}+(1 / n)\right)^{\gamma}} \varphi^{2}-2 \int_{\Omega}\left[a(x)+u_{n}^{q}\right]\left[1-\left(u_{n}+1\right)^{1-q}\right] \nabla u_{n} \nabla \varphi \varphi .
\end{aligned}
$$

Using Young's inequality with $\epsilon$, we have by (3) and Lemma 3 that

$$
\begin{aligned}
2 \mid \int_{\Omega}[a(x) & \left.+u_{n}^{q}\right]\left[1-\left(u_{n}+1\right)^{1-q}\right] \nabla u_{n} \nabla \varphi \varphi \mid \\
& \leq \varepsilon \int_{\Omega}\left|\nabla u_{n}\right|^{2} \varphi^{2}+C(\varepsilon) \int_{\Omega}|\nabla \varphi|^{2} .
\end{aligned}
$$

Taking the above estimate in (44) and letting $\varepsilon=\left(\min (1, \alpha) / 2^{q}\right)$, we obtain

$$
\frac{\min (1, \alpha)}{2^{q}} \int_{\Omega}\left|\nabla u_{n}\right|^{2} \varphi^{2} \leq \frac{\|\varphi\|_{L^{\infty}(\Omega)}^{2}}{c_{\omega}^{\gamma}} \int_{\Omega} f+C,
$$

and thus, Lemma 7 is proved.

Proof. of Theorem 1.

We start by proving point (1.i), the rest of the proof of the theorem can be proven similarly. According to Lemmas 3 and 4 , there exists a subsequence $u_{n}$ and a function $u \in H_{0}^{1}(\Omega) \cap L^{\infty}(\Omega)$ such that $u_{n}$ weakly converges to $u$ in $H_{0}^{1}(\Omega)$. Now, we can pass to the limit in the equation satisfied by the approximated solutions $u_{n}$ :

$$
\int_{\Omega}\left[a(x)+u_{n}^{q}\right] \nabla u_{n} \nabla \varphi=\int_{\Omega} \frac{f_{n} \varphi}{\left(u_{n}+(1 / n)\right)^{\gamma}}, \quad \forall \varphi \in C_{0}^{1}(\Omega),
$$

where $f_{n}(x)=(f(x) / 1+(1 / n) f(x))$.

For the term of the left-hand side, it is sufficient to observe that $\nabla u_{n}$ converge to $\nabla u$ weakly in $L_{\text {loc }}^{2}(\Omega)$ and $\left[a(x)+u_{n}^{q}\right]$ a.e. (and weakly $-{ }^{*}$ in $L^{\infty}(\Omega)$ converges towards $\left[a(x)+u^{q}\right]$. On the contrary, for the limit of the right-hand side of (47), let $\omega=\operatorname{Supp} \varphi$, and one can use Lebesgue's dominated convergence theorem, since

$$
\left|\frac{f_{n} \varphi}{\left(u_{n}+(1 / n)\right)^{\gamma}}\right| \leq \frac{f|\varphi|}{c_{\omega}^{\gamma}} .
$$

Finally, passing to the limit as $n$ goes to infinity in equation (47), we conclude that

$$
\int_{\Omega}\left[a(x)+u^{q}\right] \nabla u \nabla \varphi=\int_{\Omega} \frac{f \varphi}{u^{\gamma}}, \quad \forall \varphi \in C_{0}^{1}(\Omega) .
$$

2.2. Further Existence Result. In this section, we suppose (2) and (3) and we assume that

$$
0<q<1
$$

holds true.

Lemma 8. We suppose that (2), (3), and (50) hold true. Let $\gamma<1-q$ and $0 \leqq f \in L^{m}(\Omega)$, with

$$
\frac{2^{*}}{2^{*}+q-1+\gamma} \leq m<\frac{N}{2} .
$$

Then, the solutions $u_{n}$ to problem (19) are uniformly bounded in $H_{0}^{1}(\Omega) \cap L^{m^{* *}(1+q+\gamma)}(\Omega)$.

Proof. Let us take $\left(1+u_{n}\right)^{1-q}-1$ as a test function in (19) and use assumption (3) to obtain 


$$
\begin{aligned}
(1-q) \min (1, \alpha) \int_{\Omega}\left|\nabla u_{n}\right|^{2} & \leq(1-q) \int_{\Omega} \frac{a(x)+u_{n}^{q}}{\left(1+u_{n}\right)^{q}}\left|\nabla u_{n}\right|^{2} \\
& \leq C \int_{\Omega} f\left|u_{n}\right|^{1-q-\gamma} .
\end{aligned}
$$

We can use Hölder's inequality on the right-hand side with exponent $\quad p=\left(2^{*} / 2^{*}+q-1+\gamma\right)=(2 N$ $/ N(\gamma+1+q)+2(1-q-\gamma))>1$, and Sobolev inequality on the left-hand side to deduce

$$
\mathcal{S} \min (1, \alpha)(1-q)\left(\int_{\Omega} u_{n}^{2^{*}}\right)^{2 / 2^{*}} \leq C\left(\int_{\Omega} u_{n}^{p^{\prime}(1-q-\gamma)}\right)^{1 / p^{\prime}} .
$$

We note that $2^{*}=p^{\prime}(1-q-\gamma)$; moreover, $\left(2 / 2^{*}\right) \geq\left(1 / p^{\prime}\right)$ (thanks to the fact that $\left.\gamma<1-q\right)$. This last estimate imply that $u_{n}$ is uniformly bounded in $L^{2^{*}}(\Omega)$ and in $H_{0}^{1}(\Omega)$.

We are going to prove now that the sequence $u_{n}$ is bounded in $L^{m^{* *}(1+q+\gamma)}(\Omega)$. Let $\lambda=(N(1+q)(m-1)+\gamma m(N-2) / N-2 m) ; \quad$ using $\left(1+u_{n}\right)^{\lambda}-1$ as a test function for problem (19), we can deduce

$$
\begin{aligned}
\lambda \min (1, \alpha) \int_{\Omega} \frac{\left|\nabla u_{n}\right|^{2}}{\left(1+u_{n}\right)^{1-\lambda-q}} & \leq \int_{\Omega} \frac{f_{n}}{\left((1 / n)+u_{n}\right)^{\gamma}}\left[\left(1+u_{n}\right)^{\lambda}-1\right] \\
& \leq C+C \int_{\Omega} \frac{f}{\left(1+u_{n}\right)^{\gamma-\lambda}}
\end{aligned}
$$

Now, we rewrite

$$
\frac{4 \lambda \min (1, \alpha)}{(1+q+\lambda)^{2}} \int_{\Omega}\left|\nabla\left[\left(1+u_{n}\right)^{1+q+\lambda / 2}-1\right]\right|^{2}=\lambda \min (1, \alpha) \int_{\Omega} \frac{\left|\nabla u_{n}\right|^{2}}{\left(1+u_{n}\right)^{1-\lambda-q}}
$$

and use the Sobolev inequality and the Hölder inequality in (54) to obtain

$$
\left(\int_{\Omega}\left|\left(1+u_{n}\right)^{1+q+\lambda / 2}-1\right|^{2^{*}}\right)^{2 / 2^{*}} \leq\left(\int_{\Omega}\left|u_{n}+1\right|^{m^{\prime}(\lambda-\gamma)}\right)^{1 / m^{\prime}} .
$$

We note that the choice of $\lambda$ is equivalent to require $\left(2 / 2^{*}\right)(1+q+\lambda)=m^{\prime}(\lambda-\gamma)$ furthermore, $\left(2 / 2^{*}\right) \geq\left(1 / m^{\prime}\right)$ and $\left(2 / 2^{*}\right)(1+q+\lambda)=m^{* *}(1+q+\gamma)$. Thus, the sequence $\left\{u_{n}\right\}$ is uniformly bounded in $L^{m^{* *}(1+q+\gamma)}(\Omega)$.

Lemma 9. Under the hypotheses $0 \supsetneqq f \in L^{1}(\Omega)$, (2), (3), and (50), if $\gamma=1-q$, then the solutions $u_{n}$ are uniformly bounded in $H_{0}^{1}(\Omega)$.

Proof. We choose $\left(1+u_{n}\right)^{1-q}-1$ as test function in (19) to obtain, by hypothesis (3), that

$$
(1-q) \min (\alpha, 1) \int_{\Omega}\left|\nabla u_{n}\right|^{2} \leq(1-q) \int_{\Omega} \frac{a(x)+u_{n}^{q}}{\left(1+u_{n}\right)^{q}}\left|\nabla u_{n}\right|^{2} \leq C \int_{\Omega} f .
$$

Therefore, $u_{n}$ is bounded in $H_{0}^{1}(\Omega)$.

Lemma 10. Let $0 \lessgtr f \in L^{1}(\Omega)$. Under hypotheses (2), (3), and (50), if $\gamma>1-q$, then the solutions $u_{n}$ are uniformly bounded in $L^{(1+q+\gamma / 2) 2^{*}}(\Omega) \cap H_{l o c}^{1}(\Omega)$.

Proof. Choosing $u_{n}^{\gamma}$ as test function in (19) and using Hölder and Sobolev inequalities, thanks to (3), we obtain that

$$
\begin{aligned}
& \frac{4 \gamma \mathcal{S}}{(1+q+\gamma)^{2}}\left(\int_{\Omega} u_{n}^{(1+q+\gamma / 2) 2^{*}}\right)^{2 / 2^{*}} \leq \gamma \int_{\Omega}\left|\nabla u_{n}\right|^{2} u_{n}^{\gamma+q-1}, \\
& \quad \leq \gamma \int_{\Omega}\left[a(x)+u_{n}^{q}\right]\left|\nabla u_{n}\right|^{2} u_{n}^{\gamma-1} \leq \int_{\Omega} f .
\end{aligned}
$$

The above inequality implies that

$$
\int_{\Omega} u_{n}^{(1+q+\gamma / 2) 2^{*}} \leq C
$$

Now, we prove that the sequence $u_{n}$ is bounded in $H_{\text {loc }}^{1}(\Omega)$. Let $\varphi \in C_{0}^{1}(\Omega)$ and choose $\left[\left(1+u_{n}\right)^{1-q}-1\right] \varphi^{2}$, as a test function in problems (19). From assumption (19), one has

$$
\begin{gathered}
\min (1, \alpha) \int_{\Omega}\left|\nabla u_{n}\right|^{2} \varphi^{2}++2 \int_{\Omega}\left[a(x)+u_{n}^{q}\right]\left[\left(u_{n}+1\right)^{1-q}-1\right] \nabla u_{n} \nabla \varphi \varphi \\
\int_{\Omega} \frac{f_{n}}{\left(u_{n}+(1 / n)\right)^{\gamma}}\left[\left(u_{n}+1\right)^{1-q}-1\right] \varphi^{2} \leq \frac{\|\varphi\|_{L^{\infty}(\Omega)}^{2}}{c_{\omega}^{\gamma-q+1}} \int_{\Omega} f
\end{gathered}
$$


where $\omega=\operatorname{Supp} \varphi$. We can use Young's inequality with $\epsilon$ and both (37) and (59) to obtain

$$
\begin{aligned}
& 2\left|\int_{\Omega}\left[a(x)+u_{n}^{q}\right]\left[\left(u_{n}+1\right)^{1-q}-1\right] \nabla u_{n} \nabla \varphi \varphi\right| \\
& \leq \varepsilon \int_{\Omega}\left|\nabla u_{n}\right|^{2} \varphi^{2}+C(\varepsilon) \int_{\Omega}\left[a(x)+u_{n}^{q}\right]\left[\left(u_{n}+1\right)^{1-q}-1\right]|\nabla \varphi|^{2} \\
& \leq \varepsilon \int_{\Omega}\left|\nabla u_{n}\right|^{2} \varphi^{2}+C(\varepsilon) c_{0} \int_{\Omega} u_{n}^{2}|\nabla \varphi|^{2} \\
& \leq \varepsilon \int_{\Omega}\left|\nabla u_{n}\right|^{2} \varphi^{2}+C(\varepsilon) .
\end{aligned}
$$

Hence, equality (60) implies that $\min (1, \alpha) \int_{\Omega}\left|\nabla u_{n}\right|^{2} \varphi^{2} \leq \frac{\|\varphi\|_{L^{\infty}(\Omega)}^{2}}{c_{\omega}^{\gamma+q-1}} \int_{\Omega} f+\varepsilon \int_{\Omega}\left|\nabla u_{n}\right|^{2} \varphi^{2}+C(\varepsilon)$
Letting $\varepsilon=(\min (1, \alpha) / 2)$, we get that $u_{n}$ is bounded in $H_{\mathrm{loc}}^{1}(\Omega)$.

Lemma 11. Under the assumptions of Theorem 2, let $u_{n}$ be a solution to problem (19). Then, the sequence $u_{n}^{q}\left|\nabla u_{n}\right|$ is uniformly bounded in $L_{l o c}^{\sigma}(\Omega)$, for every $\sigma<(N / N-1)$.

Proof. We will prove our proof in two steps:

Step 1: we want to prove that, for every $\lambda>1$, $\left(1+u_{n}\right)^{q-\lambda}\left|\nabla u_{n}\right|^{2} \in L_{\text {loc }}^{1}(\Omega)$. Indeed, let $\lambda>1$, $\varphi \in C_{0}^{1}(\Omega)$ and $\omega=\operatorname{Supp} \varphi$ is the support of $\varphi$. Thanks to (3), we have from (19) with test function $\left[1-\left(1 /\left(1+u_{n}\right)^{\lambda-1}\right)\right] \varphi^{2}$

$$
(\lambda-1) \int_{\Omega} \frac{\left|\nabla u_{n}\right|^{2}}{\left(1+u_{n}\right)^{\lambda-q}} \varphi^{2}+2 \int_{\Omega}\left[a(x)+u_{n}^{q}\right]\left[1-\left(u_{n}+1\right)^{1-\lambda}\right] \nabla u_{n} \nabla \varphi \varphi \leq C(\omega)
$$

We use Young's inequality, and since $q<1$, we deduce from (37) that

$$
\begin{aligned}
(\lambda-1) \int_{\Omega} \frac{\left|\nabla u_{n}\right|^{2}}{\left(1+u_{n}\right)^{\lambda-q}} \varphi^{2} \\
\leq C(\omega)+\frac{1}{2} \int_{\Omega}\left|\nabla u_{n}\right|^{2} \varphi^{2}+\frac{1}{2} \int_{\Omega}\left[a(x)+u_{n}^{q}\right]^{2} \\
\quad\left[1-\left(u_{n}+1\right)^{1-\lambda}\right]^{2}|\nabla \varphi|^{2} \\
\leq C(\omega)+\frac{1}{2} \int_{\Omega}\left|\nabla u_{n}\right|^{2} \varphi^{2}+\frac{c_{0}}{2} \int_{\Omega}\left(1+u_{n}\right)^{2 q}|\nabla \varphi|^{2} \\
\leq C(\omega)+\frac{1}{2} \int_{\Omega}\left|\nabla u_{n}\right|^{2} \varphi^{2}+C \int_{\Omega} u_{n}^{2}|\nabla \varphi|^{2}+C .
\end{aligned}
$$

Thus, by the above estimate and since $u_{n}$ is uniformly bounded in $H_{\text {loc }}^{1}(\Omega)$, this proves Step 1 .

Step 2: here, we show that $u_{n}^{q}\left|\nabla u_{n}\right|$ is uniformly bounded in $L_{\mathrm{loc}}^{r}(\Omega)$ for every $r<(N / N t-n 1)$. For this, let $\sigma<2, \quad 0<\varphi \in C_{0}^{1}(\Omega)$, and $\omega=\operatorname{Supp} \varphi$. We use Hölder inequality with exponent $2 / \sigma$ and by step 1 , and1 we obtain

$$
\begin{aligned}
& \int_{\Omega} u_{n}^{q \sigma}\left|\nabla u_{n}\right|^{\sigma} \varphi^{\sigma} \\
& \leq \int_{\Omega} \frac{\left|\nabla u_{n}\right|^{\sigma}}{\left(1+u_{n}\right)^{\sigma(\lambda-q) / 2}} \varphi^{\sigma^{2}(N-2) / 2(N-\sigma)}\left(1+u_{n}\right)^{\sigma(\lambda+q) / 2} \varphi^{N \sigma(2-\sigma) / 2(N-\sigma)} \\
& \leq\left(\int_{\Omega} \frac{\left|\nabla u_{n}\right|^{2}}{\left(1+u_{n}\right)^{\lambda-q}} \varphi^{\sigma(N-2) / N-\sigma}\right)^{\sigma / 2}\left(\int_{\Omega}\left(1+u_{n}\right)^{\sigma(\lambda+q) / 2-\sigma} \varphi^{\sigma^{*}}\right)^{2-\sigma / 2} \\
& \leq C(\omega)\left(\int_{\Omega}\left(1+u_{n}\right)^{\sigma(\lambda+q) / 2-\sigma} \varphi^{\sigma^{*}}\right)^{2-\sigma / 2} .
\end{aligned}
$$


Using the Sobolev inequality, we obtain

$$
\begin{aligned}
\left(\int_{\Omega} u_{n}^{(q+1) \sigma^{*}} \varphi^{\sigma^{*}}\right)^{\sigma / \sigma^{*}} \leq & C(\omega)\left(\int_{\Omega}\left(1+u_{n}\right)^{\sigma(\lambda+q) / 2-\sigma} \varphi^{\sigma^{*}}\right)^{2-\sigma / 2} \\
& +C(\omega) .
\end{aligned}
$$

Noticing that $\left(\sigma / \sigma^{*}\right)>(2-\sigma / 2)$ and choosing $\sigma$ such that $(q+1) \sigma^{*}=(\sigma(\lambda+q) / 2-\sigma) \quad$ yields $\sigma=(N(2+q-\lambda) / N(q+1)-(\lambda+q))$. Using Young's inequality with $\epsilon$, we obtain

$$
\left(\int_{\Omega} u_{n}^{(q+1) \sigma^{*}} \varphi^{\sigma^{*}}\right)^{\sigma / \sigma^{*}} \leq \varepsilon\left(\int_{\Omega}\left(1+u_{n}\right)^{(q+1) \sigma^{*}} \varphi^{\sigma^{*}}\right)^{\sigma / \sigma^{*}}+C(\omega, \varepsilon) .
$$

It is easy to check that the hypotheses $\lambda>1$ imply $\sigma<(N / N-1)<2$.

\section{Proof. of Theorem 2.}

The proof of the theorem is similar to the proof of the previous theorem with just a small change for the convergence of the term on the left side of equation (47). Indeed, using Lemma 11, we have that $\left[a(x)+u_{n}^{q}\right] \nabla u_{n} \longrightarrow[a(x)+$ $\left.u^{q}\right] \nabla u$ is weak in $\left(L_{\text {loc }}^{\sigma}(\Omega)\right)^{N}$ for every $\sigma<(N / N-1)$. Hence, for every $\varphi \in C_{0}^{1}(\Omega)$, we can pass to the limit with respect to $n$ in the integral in the left-hand side of (47).

Remark 1. Assume that (2) and (3) are satisfied. We can choose $u_{n}^{\gamma}$, as test function in (19), using (3), and we obtain that

$$
\begin{aligned}
& \frac{4 \gamma}{(\gamma+q+1)^{2}} \int_{\Omega}\left|\nabla\left(u_{n}^{\gamma+q+1 / 2}\right)\right|^{2}=\gamma \int_{\Omega}\left|\nabla u_{n}\right|^{2} u_{n}^{\gamma+q-1}, \\
& \leq \gamma \int_{\Omega}\left[a(x)+u_{n}^{q}\right]\left|\nabla u_{n}\right|^{2} u_{n}^{\gamma-1} \leq \int_{\Omega} f .
\end{aligned}
$$

We deduce from (68) that the sequence $u_{n}^{\gamma+q+1 / 2}$ is bounded in $H_{0}^{1}(\Omega)$. Therefore, $u^{\gamma+q+1 / 2}$ belongs to $H_{0}^{1}(\Omega)$.

\section{Data Availability}

No data were used to support the study.

\section{Conflicts of Interest}

The authors declare that they have no conflicts of interest.

\section{References}

[1] D. Arcoya, S. Barile, and P. J. Martínez-Aparicio, "Singular quasilinear equations with quadratic growth in the gradient without sign condition," Journal of Mathematical Analysis and Applications, vol. 350, no. 1, pp. 401-408, 2009.

[2] D. Arcoya, J. Carmona, and P. J. Martínez-Aparicio, "Elliptic obstacle problems with natural growth on the gradient and singular nonlinear terms," Advanced Nonlinear Studies, vol. 7, pp. 299-317, 2007.

[3] D. Arcoya and P. Martínez-Aparicio, "Quasilinear equations with natural growth," Revista Matemática Iberoamericana, vol. 24, pp. 597-616, 2008.
[4] D. Arcoya and S. Segura De León, "Uniqueness of solutions for some elliptic equations with a quadratic gradient term," ESAIM: Control, Optimisation and Calculus of Variations, vol. 16, no. 2, pp. 327-336, 2010.

[5] S. Buccheri, "Sign-changing solutions for elliptic problems with singular gradient terms and $L^{1}(\Omega)$ data," Nonlinear Differential Equations and Applications, vol. 25, pp. 25-34, 2018.

[6] L. Boccardo, "Dirichlet problems with singular and gradient quadratic lower order terms," ESAIM: Control, Optimisation and Calculus of Variations, vol. 14, no. 3, pp. 411-426, 2008.

[7] L. Boccardo, L. Moreno-Mérida, and L. Orsina, "A class of quasilinear dirichlet problems with unbounded coefficients and singular quadratic lower order terms," Milan Journal of Mathematics, vol. 83, no. 1, pp. 157-176, 2015.

[8] A. Bouhlal, A. El Hachimi, J. Igbida, E. M. Sadek, and H. Talibi Alaoui, "Existence of solutions for unbounded elliptic equations with critical natural growth," International Journal of Differential Equations, vol. 20187 pages, 2018.

[9] D. Giachetti, F. Petitta, and S. Segura De Leon, "Elliptic equations having a singular quadratic gradient term and changing sign datum," Communications on Pure \& Applied Analysis, vol. 11, pp. 1875-1895, 2013.

[10] L. Boccardo, "A contribution to the theory of quasilinear elliptic equations and application to the minimization of integral functionals," Milan Journal of Mathematics, vol. 79, no. 1, pp. 193-206, 2011.

[11] L. Boccardo and L. Orsina, "Semilinear elliptic equations with singular nonlinearities," Calculus of Variations and Partial Differential Equations, vol. 37, no. 3-4, pp. 363-380, 2010.

[12] G. Croce, "An elliptic problem with two singularities," Asymptotic Analysis, vol. 78, no. 1-2, pp. 1-10, 2012.

[13] J. Leray and J.-L. Lions, "Quelques résultats de Višik sur les problèmes elliptiques non linéaires par les méthodes de Minty-Browder," Bulletin de la Société mathématique de France, vol. 79, pp. 97-107, 1965.

[14] G. Stampacchia, "Le problème de Dirichlet pour les équations elliptiques du second ordre à coefficients discontinus," Annales de l'institut Fourier, vol. 15, no. 1, pp. 189-257, 1965. 